\title{
Dermatologische Laboratoriumsdiagnostik - wissenschaftlich begründet, praxisrelevant und wirtschaftlich. 8. Leipziger Labor-Workshop 2009
}

\author{
Dermatologic Laboratory Diagnostics - Scientifically Justified, Relevant for Practice, and Economically. \\ 8th Leipzig Laboratory Workshop 2009 - Report from the Meeting
}

Autoren

Institute
P. Nenoff', G. Hamm²

Laboratorium für medizinische Mikrobiologie, Mölbis

Hautarztpraxis, Halle (Saale)

\section{Bibliografie}

Dol http://dx.doi.org/ 10.1055/s-0029-1214948

Online-Publikation: 20.7. 2009

Akt Dermatol 2009; 35:

515-521 ๑ Georg Thieme

Verlag KG Stuttgart · New York ISSN 0340-2541

\section{Korrespondenzadresse}

Prof. Dr. med. Pietro Nenoff Haut- und Laborarzt/

Allergologie, Andrologie

Laboratorium für medizinische Mikrobiologie

Straße des Friedens 8

04579 Mölbis

nenoff@mykologie-experten.de

\section{Einleitung \\ $\nabla$}

Am 10. Januar 2009 fand in Leipzig der mittlerweile 8. Workshop „Dermatologische Laboratoriumsdiagnostik - wissenschaftlich begründet, praxisrelevant und wirtschaftlich" als Veranstaltung des Berufsverbandes Deutscher Dermatologen statt ( Abb. 1). Die von Prof. Dr. Uwe-Frithjof Haustein, Leipzig, moderierte Tagung wird bestens akzeptiert, was sich in der gestiegenen Teilnehmerzahl von fast 90 Hautärzten - ambulant und z.T. auch stationär tätig - aus ganz Deutschland widerspiegelt.

\section{Qualitätssicherung bei Labor- untersuchungen in der Arztpraxis - Rili-BÄK 2008 \\ $\nabla$ \\ Dr. rer. nat. Friedrich Brune, Magdeburg}

Seit dem 1.4.2008 ist die neue „Richtlinie der Bundesärztekammer zur Qualitätssicherung labormedizinischer Untersuchungen“ in Kraft. Sie wurde nach mehrjähriger Vorbereitung am 23. 11. 2007 in der Bundesärztekammer beschlossen.

Gegenüber der alten Richtlinie vom 24.8.2001 sind neue Inhalte hinzugekommen, die von jedem beachtet werden müssen, der in der Heilkunde Laboruntersuchungen vornimmt. Im Gegensatz zur alten Rili-BÄK beschränkt sich die neue Richtlinie nicht nur auf quantitative Analysen, sondern gilt für alle Laboruntersuchungen!

Diese Richtlinie unterteilt sich in die Teile A bis G. Für das Anwenden in der Praxis sind zunächst die Teile A und B wichtig. Es gilt eine Übergangszeit von 2 Jahren. In dieser Zeit kann intern und extern nach der alten Richtlinie gearbeitet werden. Ringversuche werden allerdings ab 1.1.2009 nach der neuen Rili-BÄK bewertet.

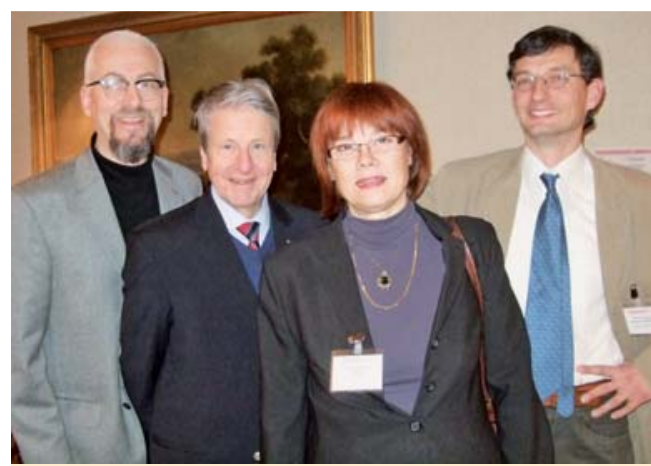

Abb. 1 Referenten und Organisatoren des Leipziger Labor-Workshops 2009: Priv.-Doz. Jörg Kleine-Tebbe (Berlin), Prof. Uwe-Frithjof Haustein (Leipzig), Dr. Gudrun Hamm (Halle/Saale) und Prof. Pietro Nenoff (Mölbis), (von links nach rechts).

Teil A gilt für ausnahmslos alle Laboruntersuchungen. Dieser Teil verlangt neben prä- und postanalytischen Prozessen das genaue Beschreiben aller Laborprozesse. Teil A ist angefüllt mit anstrengenden Aufgaben, die vom Anwender Punkt für Punkt abzuarbeiten sind. Es ist ein detailliertes Qualitätsmanagementsystem auszuarbeiten, welches u.a. für jede Laboruntersuchung eine genaue Verfahrensanweisung verlangt. Teil A enthält auch 43 konkrete Definitionen grundlegender Sachverhalte.

Teil B behandelt die speziellen Laboruntersuchungen. Bisher ist der Teil B1 erschienen, der quantitative Laboruntersuchungen enthält. In nachfolgenden Kapiteln (B2 bis Bx) werden weitere spezielle Untersuchungen folgen (z. B. qualitative Untersuchungen, Untersuchungen von Krankheitserregern u.a.).

Die Qualitätskontrolle quantitativer Untersuchungen (B 1) ist komplett überarbeitet worden. Die interne Qualitätskontrolle ist für alle quantitativen Untersuchungen verbindlich. Die zulässigen Fehlergrenzen sind in der Tabelle B1 a - c niedergelegt. Zentrale Begriffe bei der internen Qua- 
litätskontrolle sind die relative Messabweichung und der quadratische Mittelwert der Messabweichung. Neu ist, dass diese Abweichungen aus jedem Kontrollwert und dem Zielwert der Kontrolle errechnet werden. Daher hat der Zielwert der Kontrollprobe eine eminente Bedeutung. Laborinterne Fehlergrenzen sind nur bei den Parametern erforderlich, die nicht in der Tabelle B1 a - c enthalten sind.

Die patientennahe Sofortdiagnostik ist klar definiert (keine Probenvorbereitung, Einzelprobenmessung, sofortige Ableitung therapeutischer Konsequenzen). Von der neuen Rili-BÄK werden nur noch solche Geräte favorisiert, die Unit-use-Reagenzien verwenden. Das sind solche Reagenziensysteme, die mit einer Untersuchung verbraucht sind. Außerdem sollen diese Geräte physikalische oder elektronische Standards anwenden und so, oder durch eine andere integrierte Prüfung der Gerätefunktion, verhindern, dass fehlerhafte Messergebnisse ausgegeben werden können. Nur unter diesen Voraussetzungen ist die vereinfachte Qualitätskontrolle im bisherigen Umfang ausreichend.

Die externe Qualitätskontrolle (Ringversuche) ist für alle quantitativen Untersuchungen verbindlich, die in der Tabelle B1 a-c aufgeführt sind. Wie bisher ist ein Ringversuch pro Quartal gefordert.

\section{Streptokokkenerkrankungen der Haut \\ $\nabla$}

\section{Prof. Dr. med. Cord Sunderkötter, Münster}

Die wichtigsten humanpathogenen Arten der Streptokokken sind $\beta$-hämolysierende Streptokokken der Gruppe A (S. pyogenes). Zu den häufiger von ihnen verursachten Infektionen gehören das Erysipel und im Kindesalter die Impetigo contagiosa. Das klassische, durch $\beta$-hämolysierende Streptokokken verursachte Erysipel wird klinisch diagnostiziert anhand der Symptome überwärmtes hellrotes Erythem oder nur leicht erhabene erythematöser Plaque, glänzende Oberfläche, scharf begrenzte Ränder und charakteristische zungenförmige Ausläufer als Folge der Infektionsausbreitung entlang der Lymphspalten und Lymphgefäße. Es sollte differenzialdiagnostisch von einer Reihe anderer Erkrankungen unterschieden werden. Darunter befinden sich die akute Stauungsdermatitis (nicht selten als Erysipel fehldiagnostiziert) und die Weichteilinfektion (Zellulitis bzw. Phlegmone). Sie werden häufig durch $S$. aureus, oder auch andere Bakterien verursacht, und haben als klinische Zeichen einen rotlividen Farbton, matte Oberfläche, teigige ödematöse Schwellung und oft Ausgang von Ulzera (sie werden im Ggs. zum Erysipel nicht mit Penicillin G oder V, sondern mit Penicillinase-festen oder breiter wirkenden Antibiotika behandelt).

Weitere Streptokokken-bedingte Infektionen sind das Ekthyma mit seinen ausgestanzten Ulzera sowie die oft schmerzhafte und mitunter nicht erkannte perianale Zellulitis. Unter bestimmten, den Erreger und das Immunsystem betreffenden Voraussetzungen verursachen $\beta$-hämolysierende Streptokokken die lebensbedrohliche nekrotisierende Fasziitis die mit Zeichen eines Streptococcal Toxic Shock Syndrome (STSS) einhergeht.

Wenn in Streptokokken die Gene für das pyrogene Toxin A oder C vorhanden sind, kommt es im Rahmen einer Streptokoken-Infektion zum Scharlach. Heute selten ist die Postreptokokken-Erkrankung Glomerulonephritis, die aber routinemäßig durch eine einfache Urinuntersuchung 10-20 Tage nach Infektion ausgeschlossen werden kann. Mittel der Wahl bei Infektionen mit Streptokokken sind Penicillin V oder G.

\section{Allergologische In vitro-Diagnostik in Europa: Auswirkungen auf die deutsche Hautarztpraxis?} $\boldsymbol{\nabla}$

\author{
PD Dr. med. Jörg Kleine-Tebbe, Berlin
}

In den vergangenen 20 Jahren ist ein enormer Fortschritt bei der Entdeckung neuer Allergene zu verzeichnen. Damit sind die verantwortlichen Proteine gemeint - also Allergene im engeren Sinne -, die für die Reaktionen gegenüber unterschiedlichen Allergenquellen (z.B. Pollen, Milben, Tierepithelien, Schimmelpilze, Insektengifte) verantwortlich sind. Mit offizieller WHO-Nomenklatur versehen, wurden diese Allergene in die offizielle Allergen-Datenbank aufgenommen (nach biologischen Stammbäumen katalogisiert: www.allergen.org/Allergen.aspx), die abgesehen von ihrer spezifischen Suchfunktion ohne Kenntnisse der biologischen Nomenklatur wenig Nutzen bietet. Eine andere umfangreiche englischsprachige Allergen-Datenbank ist mit zusätzlichen Suchfunktionen, Verknüpfungen, Informationen und Literaturstellen versehen (www.allergome.org). Aufgrund ihrer Strukturähnlichkeit können die identifizierten Allergene definierten Proteinfamilien zugeordnet werden, die im Internet einschließlich ihrer Verwandten gelistet und mit anderen Datenbanken verknüpft wurden (http://www.meduniwien.ac.at/allergens/ allfam/). Überraschenderweise sind es trotz der enormen Vielfalt an nicht-humanen Proteinen in der belebten Umwelt nur wenige Vertreter, die eine allergische Sensibilisierung und anschließend Symptome auslösen. Wahrscheinlich bilden die Proteine in anderen Organismen, sofern sie auch im Menschen vorkommen, mit abnehmender Stukturähnlichkeit aufgrund ihrer evolutionärer Distanz zum „humanen Vertreter“ ein erhöhtes Risiko für eine allergische Sensibilisierung. Kurz gesagt: Um so ähnlicher ein Protein seinem humanen Vertreter ist, desto wahrscheinlicher besteht immunologische Toleranz. Um so weniger Ähnlichkeit durch entsprechende evolutionäre Distanz vorliegt, desto eher können diese Proteine zum Allergen werden.

Durch ihre gezielte Aufreinigung oder Herstellung in Fremdorganismen (rekombinante Proteine) wie z.B. Bakterien stehen viele der identifizierten Allergene mittlerweile zur In-vitro-Diagnostik zur Verfügung. Ihr Zusatz zu den bisher üblichen Extrakten aus bestimmten Allergen-Quellen kann die Empfindlichkeit derartig angereicherter („spiked“) In-vitro-Testreagenzien deutlich erhöhen. Eine Mischung der wichtigsten Einzel-Allergene kann einen Allergen-Extrakt, je nach Zusammensetzung, ersetzen und Vorteile bieten, besonders wenn einige Komponenten im Extrakt nicht ausreichend vertreten oder instabil sind. Schließlich können Einzel-Allergenkomponenten zur gezielten Diagnostik eingesetzt werden (sog. Component-Resolved-Diagnostic, CRD).

Damit können Allergenquellen (z.B. Gräserpollen-spezifische Leitallergene) von so genannten Pan-Allergenen (z.B. Profiline, Ca++-bindende Proteine) unterschieden werden, die in vielen Organismen vorkommen und für Kreuzreaktionen verantwortlich sind, die die Allergiediagnostik erschweren.

Parallel zur Allergenforschung wurden in den vergangenen Jahren zunehmend diagnostische Systeme in kleineren Dimensionen entwickelt. Mithilfe so genannter Microarrays (Glasobjektträger mit Testreagenzien in stark verkleinertem Maßstab, auch als Mikro-Chips bezeichnet), können über 100 Allergene nach dem Prinzip herkömmlicher Immunassays zur IgE-Bestimmung auf einem Quadratzentimeter getestet werden. Nach Bindung des spezifischen IgE wird dieses durch markierte Anti-IgE-Antikörper mithilfe eines Laser-Scanners erfasst und semiquantitativ ausgewertet. Durch die zusätzlichen, seit 2008 eingeführten und klinisch 
bereits gut charakterisierten Einzelallergene wird die diagnostische Qualität deutlich aufgewertet, wenngleich die derzeitige Zusammensetzung des Microarrays (ImmunoCAP ISAC ${ }^{\circledR}$, www.vbcgenomics.at) noch nicht alle Allergenquellen bereithält, die in der Routine z. B. mit Hauttests geprüft werden können.

Hinsichtlich der zugrundeliegenden analytischen Empfindlichkeit und Übereinstimmung mit anderen Immunoassays sind die Daten vielversprechend, obwohl die Reproduzierbarkeit (Abweichungen zwischen wiederholten Messungen: ImmunoCAP ISAC ${ }^{\circledR}$ Variationskoeffizienten VK zwischen 10 und 20\%) nicht das niedrige Niveau etablierter Immunassays erreicht (z. B. Phadia ImmunoCAP, $\mathrm{VK}<10 \%$ ).

Vorläufige Vergleichsuntersuchungen mit anderen diagnostischen Methoden zeigten für bestimmte Allergenquellen (Birkenund Gräserpollen, Katzenepithelien) gute Übereinstimmung, für andere eine mäßige (Hausstaubmilben) bzw. manche Allergenquellen (Beifußpollen) möglicherweise aufgrund fehlender Allergenkomponenten eine nur unzureichende Assoziation.

Die umfangreichste Erfahrung in der Micro-Chip-Diagnostik besteht in der Abteilung des italienischen Allergologen und Laborleiters Adriano Mari. Mehr als 30000 Seren wurden von dem Begründer der Datenbank „www.allergome.org“ bereits untersucht, sodass in seinem Institut enorme Erfahrung mit der Auswertung und Beratung von betroffenen Allergikern vorhanden ist. Innerhalb der nächsten Jahre werden sicherlich die bestehenden Lücken im Allergenspektrum durch Nachklonierung der verantwortlichen Allergen-Proteine geschlossen werden. Bei einer Auswertung von global gesammelten klinischen Daten in Verbindung mit den diagnostischen Resultaten wird sich möglicherweise die klinische Charakterisierung der neuen Protein-Allergene unter Zuhilfenahme geeigneter Datenbanken enorm beschleunigen lassen.

Damit steht die Allergie-Diagnostik vor neuen Herausforderungen. Während bisher nach einer ausführlichen Anamnese und körperlichen Untersuchung der betroffenen Allergiker ein Screening mithilfe von Prick-Hauttests vorgenommen worden ist, die spezifische IgE-Bestimmung als In-vitro-Methode gezielt eingesetzt und in Zweifelsfällen zur Klärung der klinischen Relevanz Provokations-Methoden bemüht wurden, wäre zukünftig ein anderes Vorgehen denkbar. Nach einem Massen-Screening gegenüber den wichtigsten Soforttyp-Allergenkomponenten aus verschiedenen Quellen (Aero- und Nahrungsmittel-Allergene, Insektengift-Allergene) könnte eine anschließende, gründliche Anamnese die klinische Relevanz der erhobenen Sensibilisierungen prüfen. Ein Hauttest wäre nur noch für Allergenquellen (Extrakte) erforderlich, die nicht mithilfe eines IgE-Screenings erfasst werden können oder deren kutane Sensibilisierung zusätzlich notwendige, diagnostische Informationen verspricht. Provokationen würden weiterhin zur klinischen Relevanzprüfung ihre Berechtigung behalten.

Potenziell könnte eine derartige Diagnostik breit gefächerte, individuelle Sensibilisierungs-Muster erfassen, spezielle RisikoMoleküle identifizieren und bei unvermuteten, spezifischen IgEErgebnissen möglicherweise die Aufklärung unklarer allergischer/anaphylaktischer Ereignisse beschleunigen. Auf der anderen Seite besteht die Gefahr, dass zahlreiche allergische Sensibilisierungen ohne korrespondierende Symptome, d.h. ohne klinische Relevanz, die Interpretation erschweren. Das gilt besonders für Proteine von Allergen-Quellen, bei denen sich eine Provokation verbietet (z.B. bei schwerer anaphylaktischer Reaktion durch ein Allergen) oder aufgrund von fehlenden Provokations- materialien (z.B. Anasakis simplex als parasitischer Wurm in Fischen) nicht machbar ist.

Für wen wäre diese neue Form einer molekularen Allergie-Diagnostik besonders interessant?

Sicher sind polysensibilisierte Patienten mit wahrscheinlicher Beteiligung kreuzreaktiver Pan-Allergene, Nahrungsmittel-Allergiker mit komplexen Sensibilisierungsmustern, Betroffene mit ungeklärten anaphylaktischen Reaktionen und Patienten mit ungewöhnlichen Sensibilisierungsmustern vielversprechende Kandidaten für diese neue Form der Allergie-Diagnostik. Bisher bildet sich dieser diagnostische Test nicht in den Ziffern der ärztlichen Gebührenordnungen ab. Die Ergebnisse der klinischen Evaluation werden darüber entscheiden, ob diese Form der „mikronisierten“ molekularen IgE-Diagnostik zukünftig ihr ganzes Potenzial entfalten kann. Allerdings sind Interpretation und die damit verknüpfte Beratungsleistung allergologisch anspruchsvoll und bedürfen erheblicher Fortbildungsanstrengungen auf dem jungen Gebiet der molekularen Allergologie.

\section{Hygiene in der Arztpraxis - Standards und Realisierung im Alltag $\nabla$ Dr. rer. nat. Silvia Fanghänel, Halle/S.}

Hygienestandards sind notwendig, um präventiv gegen nosokomiale Infektionen, hervorgerufen vor allem durch Bakterien und Viren, vorgehen zu können. Innerbetriebliche Verfahrensweisen zur Infektionshygiene müssen nach §36 des Infektionsschutzgesetzes in jeder medizinischen Einrichtung festgelegt werden. Der erforderliche Standard ist das Vorhalten eines an die jeweilige Einrichtung bzw. Arztpraxis angepassten Hygieneplanes, entsprechend den Empfehlungen des Robert-Koch-Instituts und anderer Fachgremien. Hierbei können auch spezifische Kapitel, wie der Umgang mit Patienten, die mit multiresistenten Erregern besiedelt oder infiziert sind, geregelt werden.

Vor dem Hintergrund, dass sich die multiresistenten Erreger, wie MRSA und ESBL, flächendeckend ausbreiten und zunehmend auch im ambulanten Bereich anzutreffen sind, erreicht diese Maßnahme eine hohe Priorität. Am Beispiel von MRSA und ESBL werden präventive Maßnahmen der Infektionshygiene aufgezeigt.

Explizit wird die Empfehlung des RKI zur Kontrolle von MRSA in Krankenhäusern und anderen medizinischen Einrichtungen referiert. Wesentliche Aspekte der Empfehlung, wie Isolierung von MRSA-Patienten, Schutzausrüstung des Personals, effiziente Händehygiene, Desinfektionsmaßnahmen von Flächen und Instrumenten, Entsorgung sowie Sanierungsmaßnahmen von MRSA-Trägern, sollten im stationären wie im ambulanten Bereich umgesetzt werden.

Zur Umsetzung der RKI-Empfehlung in der Arztpraxis bietet sich folgende Vorgehensweise an:

- mit entsprechendem Zeitmanagement Kontakte von MRSAPatienten zu anderen Patienten minimieren oder verhindern

- Voraussetzungen zur optimalen Händehygiene schaffen, wie z.B. der Verzicht auf Ringe und Schmuck

- nur unmittelbar benötigte Materialien und Geräte zur Behandlung von MRSA-Patienten offen bereitstellen; damit bleiben die anschließenden notwendigen Desinfektionsmaßnahmen in einem vertretbaren Aufwand

- Desinfektion aller Kontaktflächen nach Behandlungsende 

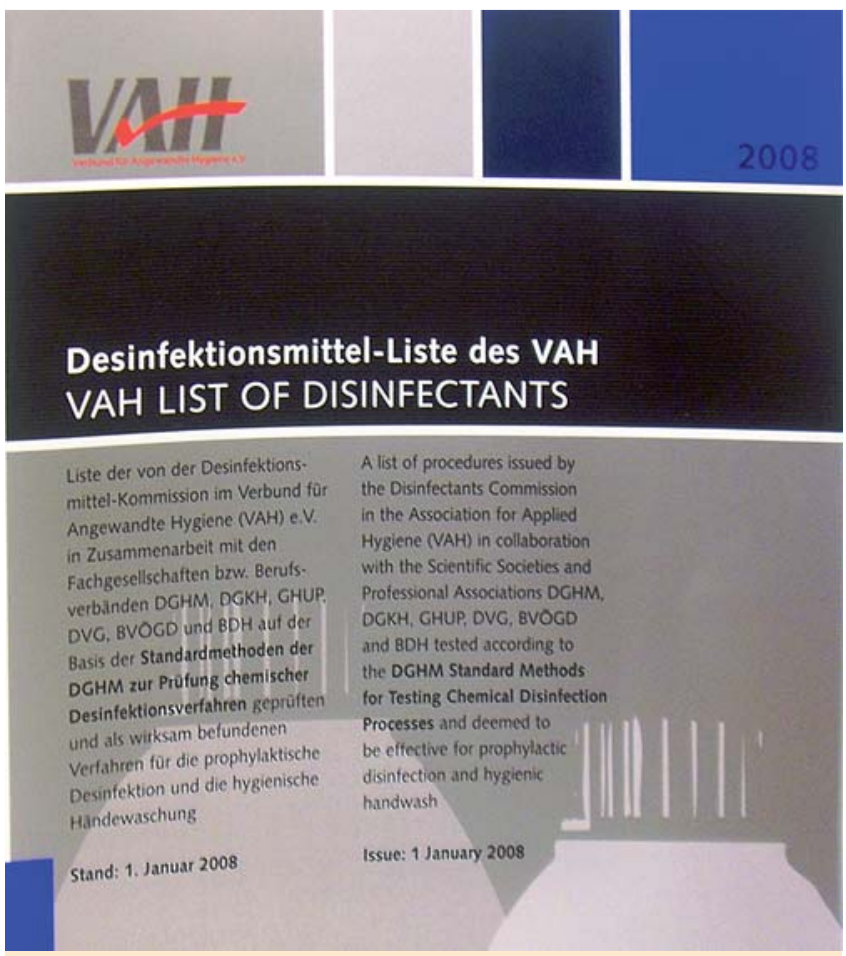

Abb. 2 Liste der Desinfektionsmittel des VAH (Verbund für Angewandte Hygiene).

- Patienten, die in einer Sanierungsphase sind, ausreichend über alle notwendigen begleitenden Maßnahmen, wie täglicher kompletter Wäschewechsel, informieren, um eine Rekolonisierung zu verhindern

- Information an weiterbehandelnde Ärzte

Die Verwendung VAH (Verbund für Angewandte Hygiene) gelisteter Produkte für sämtliche Desinfektionsmaßnahmen garantiert bei sachgerechter Anwendung Wirksamkeit. Alle in dieser Liste geführten Produkte sind mit in Deutschland anerkannten Verfahren zertifiziert $(\bullet$ Abb. 2).

Die gleichen Aspekte treffen auch beim Umgang mit ESBL-Patienten zu. Ein wesentlicher Unterschied zwischen MRSA- und ESBLPatienten besteht darin, dass es für ESBL-Patienten keine Möglichkeit der Sanierung gibt. Eine ESBL-Infektion muss mit den wenigen zur Verfügung stehenden wirksamen Antibiotika therapiert werden. Im Falle einer Besiedlung können nur adäquate Hygienemaßnahmen in der Arztpraxis die Entstehung von Infektketten verhindern.

\section{Autoimmunphänomene beim Einsatz von „Biologics“ $\nabla$ \\ Dr. med. Gudrun Hamm, Halle}

Seit etwa 20 Jahren werden so genannte „Biologics“ zur Behandlung schwerer und schwerster Verläufe bei bösartigen Neubildungen, Organtransplantationen und chronisch-entzündlichen Erkrankungen eingesetzt. Seit etwa 5 Jahren ist diese völlig neuartige Wirkstoffgruppe auch den vertragsärztlich tätigen Dermatologen zugänglich.

Die Behandlung von mehreren Millionen Patienten weltweit erlaubt inzwischen recht gut, die Wirkungen, Nebenwirkungen und Risiken einer Biologikatherapie einzuschätzen. Der Vortrag reflektiert im Rahmen einer Literaturübersicht insbesondere die beim Einsatz von „Biologics“ zu beobachtenden Autoimmunphänomene.

Aktuell existieren mehr als 80 verschiedene Biologika, die zu therapeutischen Zwecken entwickelt und zugelassen wurden oder vor der Zulassung stehen. Es handelt sich um monoklonale Antikörper, Zytokine oder Fusionsproteine, die ihre Wirkung durch Neutralisierung (z.B. von proinflammatorischen Zytokinen), durch Bindung an Rezeptoren oder durch zytotoxische Vorgänge entfalten.

Biochemisch sind Biologika exogen zugeführte Glykoproteine, die nicht nur direkt immunologische Vorgänge beeinflussen, sondern selbst „Immunogene“ darstellen. Insofern ist es interessant, aber auch nicht überraschend, dass bei den bisher eingesetzten Biologika zwei immunologische Nebenwirkungen beobachtet werden können:

1. Bei bestimmten Medikamenten können Antikörper (AK) gegen den Wirkstoff selbst gebildet werden („Wirkstoff-AK“). Bei den antipsoriatisch wirksamen Biologika scheint sicher, dass Efalizumab keinerlei AK-Bildung hervorruft. Immunologisch interessant sind die TNF-alpha-Antagonisten, wobei sich die drei derzeit zugelassenen Medikamente unterschiedlich verhalten. AK gegen Etanercept und Adalimumab wurden bisher selten nachgewiesen und führten zu keinerlei Wirkverlust. Spezifische Anti-Infliximab-AK sind dagegen häufig beschrieben und gehen mit Wirkverlust einher. Infliximab-AK entwickeln sich oft nach den ersten Infusionen und sind für die stärkeren Infusionsreaktionen verantwortlich.

Das Zytokin IFN-alpha, das z.B. beim metastasierenden Melanom eingesetzt wird, induziert offensichtlich keine antiInterferon-Auto-AK, aber AK gegen Rituximab (einem antiCD20-AK, der in der Lymphombehandlung zugelassen ist) werden in der Literatur erwähnt.

2. Es werden auto-immunologische Prozesse initiiert, weil auch die „klassischen“ Auto-AK (z.B. ANA oder ds-DNS-AK) nachweisbar sind.

Sowohl ANAs unterschiedlicher Spezifität als auch organspezifische SD-AK sind bei Melanom-Patienten unter ZytokinTherapie mit IFN-alpha beschrieben worden.

In der Psoriasistherapie lassen sich unter anti-TNF-alpha-Behandlung - insbesondere unter Infliximab - bei bis zu 2/3 der Patienten eindeutig positive ANA z.T. in höheren Titern und bei ca. der Hälfte der behandelten Patienten sogar ds-DNS AK nachweisen. Die pathogenetische Bedeutung dieser Immunphänomene ist derzeit ungeklärt. Eine genauere Bewertung ist sicher erst durch ein langfristiges systematisches Erfassen dieser Parameter zu erwarten.

\section{MALDI-TOF MS als neue Methode der Identifizierung dermatologisch relevanter Pilze \\ $\nabla$}

\section{Dr. Marcel Erhard, Potsdam/Golm}

Für eine schnelle und effektive Therapie von dermatologischen Infektionskrankheiten spielt die schnelle und sichere Identifizierung der Erreger eine wichtige Rolle. Die allgemeinen Trends der Mikrobiologie ermöglichen eine deutliche Reduzierung der Analyse- und Wachstumszeiten der Erreger bei einer Qualitätserhöhung der Analyseergebnisse. So zieht neben den bekannten, auf PCR basierenden Techniken auch die MALDI-TOF MS in den Laboralltag ein. 

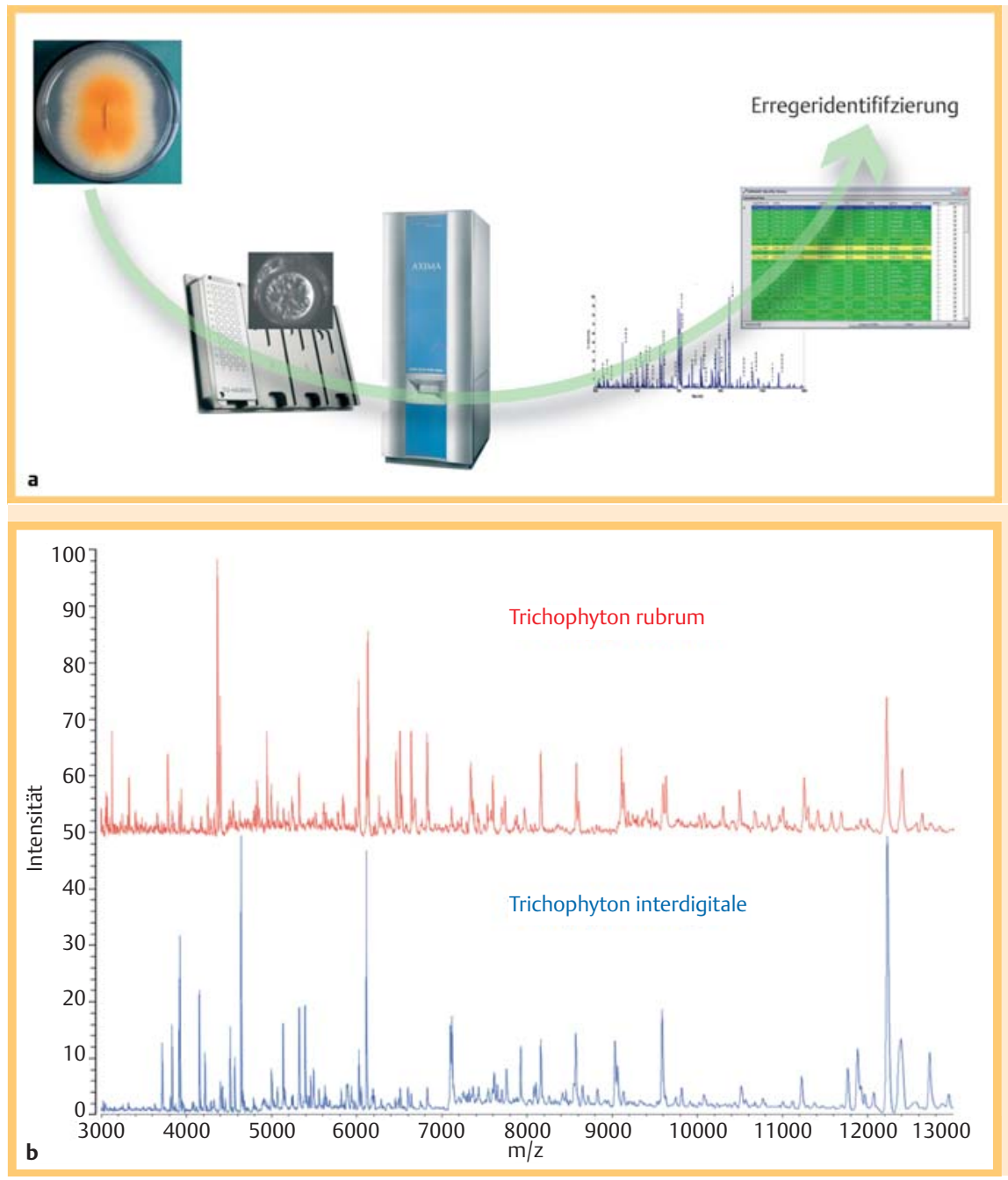

Abb. 3 a Allgemeiner Prozessablauf der AXMIA@SARAMIS MALDI-TOF MS-Identifizierung der Bakterien und Pilze.

b MALDI-TOF-Massenspektren von Trichophyton rubrum und Trichophyton interdigitale, Identifizierung von 7 Tage alten Kulturen auf Sabouraud $4 \%$-Glukose-Agar.
MALDI-TOF MS: Bei der Matrix-unterstützten Laser-Desorptionund -Ionisierung-Flugzeit-Massenspektrometrie (Matrix Assisted Laser Desorption/Ionization Time-Of-Flight Mass Spectrometry) werden die Molekulargewichte der Zellkomponenten (meist Proteine) bestimmt. Bei dieser Identifizierungsmethode ist heute noch eine kurze Kultivierung des Erregers auf Nährböden notwendig. Derzeit ist eine MALDI-TOF MS-Analyse der Erreger direkt aus Nagel- oder Hautmaterial (noch) nicht möglich. Sobald sich jedoch sichtbares Myzel gebildet hat, kann die Analyse

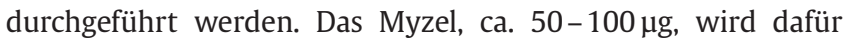
vom Nährboden abgenommen und auf den MALDI-TOF MS-Probenträger aufgetragen. In einem zweiten Schritt wird die Matrix, gelöst in einem organischen Lösungsmittel, hinzugegeben. Diese Matrixlösung dringt in die Pilzzellen ein und extrahiert die molekularen Bestandteile der Zelle. Während das Lösungsmittel verdunstet, bildet die Matrix Kristalle aus, in die auch Moleküle aus den Pilzzellen eingebaut werden. Im Massenspektrometer werden die Matrixkristalle durch Laserbeschuss desorbiert und die Moleküle ionisiert. Die dabei entstehenden Proteinionen werden in einem elektromagnetischen Feld beschleunigt und erreichen nach einer freien Flugstrecke von ca. 1,5 Meter den Detektor. Die Flugzeit der Moleküle ist proportional zu ihrer Masse, wodurch nach einer Kalibrierung des Systems mit bekannten Massen den gemessenen Peaks in einer Probe eine genaue Masse zugeordnet werden kann ( Abb. 3a). Durch die Frequenz der Laserpulse bei modernen Geräten erhält man nach nur ca. 30 Sekunden einen sog. Protein-Massen-Fingerprintabdruck der Probe. Bei Pilzen weisen die Massenspektren ca. 150 bis 200 Proteinmassen auf, die für den Vergleich mit Referenzspektren herangezogen werden können.

SARAMIS-Auswertung und -Identifizierung: Die Massenspektren der Proben werden in die SARAMIS-Software und -Datenbank (Spectral ARchive And Microbial Identifikation System) übermittelt und ausgewertet. Protein-Massen-Fingerprintabdrücke sind charakteristisch für einzelne Erregerspezies, wodurch alle Erreger identifiziert werden können, für die in der Datenbank Referenzspektren hinterlegt sind ( $\mathbf{A b b} \mathbf{3} \mathbf{3 b}$ ). Die MALDI-TOF MSReferenzspektren-Datenbank wird seit ca. 5 Jahren in einem großen Netzwerk von Partnern aufgebaut und deckt inzwischen alle typischen und relevanten Erreger ab. Erfolgt mit SARAMIS keine Identifizierung, handelt es sich im Allgemeinen um einen atypischen Vertreter einer Spezies oder um eine Spezies, für die noch kein Referenzspektrum vorliegt. Für viele Spezies ist auch eine Typisierung unterhalb der Spezies-Ebene möglich, zum Teil bis hin zur Unterscheidung von Stämmen, wodurch einzelne Epidemiestämme direkt identifiziert werden können. Mit der SARAMIS-Datenbank werden automatisch die neuesten taxonomischen Begriffe und Einteilungen der Erreger verwendet. 
Direktnachweis von Dermatophyten aus Patientenmaterial mittels Polymerasekettenreaktion (PCR) ergänzende oder alleinige diagnostische Methode in der Dermatomykologie?

$\nabla$

Pietro Nenoff, Jürgen Herrmann, Iwona Winter, Constanze Krüger, Claudia Mügge, Guntram Bezold, Silke Uhrlaß,Mölbis, Oschatz, Leipzig, Neu-Ulm

Zum Direktnachweis von Dermatophyten aus Nagelmaterial und Hautschuppen wurde ein PCR-Elisa-Assay eingesetzt ( $\bullet$ Abb. 4). Als spezifische Sequenz für die Primer wurde das Topoisomerase II-Gen genutzt. Für die DNS-Isolierung kam der Qiagen-QIAampDNA-Mini-Kit-250 zum Einsatz. Ein Primer war Digoxigeninmarkiert. Nach Amplifikation wurde das PCR-Produkt zur Visualisierung mittels biotinylisierter Sonde hybridisiert, dann an eine Streptavidin-beschichtete Festphase gebunden. Nach Zugabe eines Peroxidase-konjugierten Anti-Digoxigenin-Antikörpers sowie eines Substrates zeigte die Farbentwicklung im Elisa die positive Reaktion an. Der Uniplex-PCR-Elisa-Test erfasste separat Trichophyton (T.) rubrum, T. interdigitale und Epidermophyton floccosum - auf diese drei Dermatophyten wurden Nagelspäne untersucht, Hautschuppen zusätzlich auf Microsporum canisDNS. Die PCR wurde mit der Empfindlichkeit des fluoreszenzoptischen Nativpräparates und der kulturellen Untersuchung verglichen. In 3664 Proben (Untersuchungszeitraum 10 Monate) waren Dermatophyten mittels Kultur u./o. PCR 1414-mal nachweisbar. 960 (68\%) waren Kultur- und PCR-positiv, 201 (14\%) Proben waren Kultur-positiv, jedoch PCR-negativ, und in 253 Proben (18\%) war die Kultur negativ, trotzdem ließ sich mittels PCR ein Dermatophyt nachweisen. Die diagnostische Empfindlichkeit des Calcofluor-Präparates betrug im Vergleich zur Kultur 80,1\%, die Spezifität 80,6\%. Die diagnostische Sensitivität der Kultur auf Dermatophyten lag bei $82,1 \%$, die Spezifität - da es prinzipiell keine falsch-positiven Kulturergebnisse auf Dermatophyten geben kann - bei 100\%. Mit 85,8\% war die Sensitivität der PCR im Vergleich zur Kultur höher. Folgende Aufteilung der 1414 Dermatophyten fand sich: $68,8 \%$ T. rubrum, $20,1 \%$ T. interdigitale, $0,8 \%$ Epidermophyton floccosum, 0,3\% Microsporum canis.

Zusammenfassend kann man feststellen, dass die PCR auf Dermatophyten im Moment die klassische Diagnostik - Nativpräparat und Kultur - ergänzt und den Anteil positiver Ergebnisse erhöht, im positiven Fall wird die Zeit bis zur Diagnosestellung deutlich

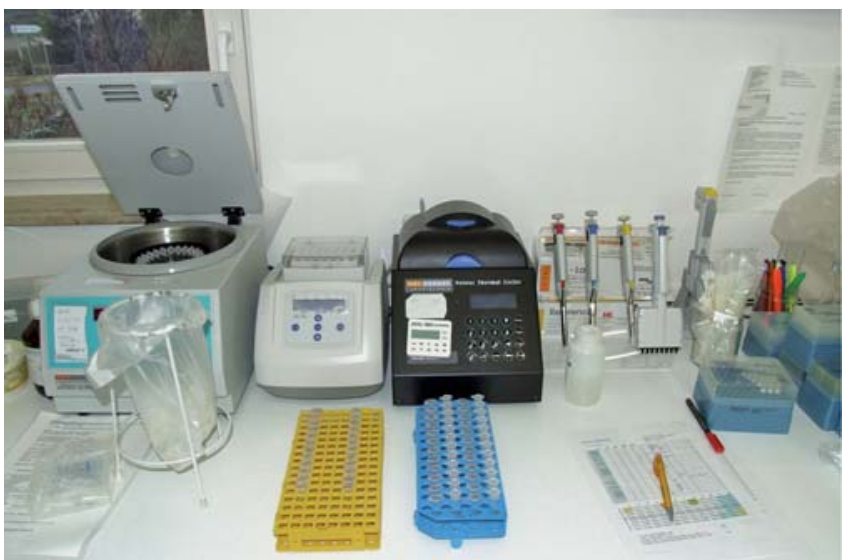

Abb. 4 PCR-Arbeitsplatz zum Nachweis von Dermatophyten-DNS aus Hautschuppen und Nagelspänen: Zentrifuge, Thermoschüttler und Thermocycler als essenzielle Minimalausstattung. verkürzt. Die Methode ist wirtschaftlich in Bezug auf die Investitionskosten und Verbrauchsmaterialien, nur der relativ hohe Personalaufwand ist limitierend. Mittel- und langfristig muss davon ausgegangen werden, dass die konventionellen Labormethoden der Dermatomykologie von molekularen Techniken abgelöst werden.

\section{Tropische Dermatosen: Erfahrungsbericht nach 29 tropenmedizinischen Fortbildungsreisen für Ärzte nach Kenia, Uganda und Tansania (seit 1995) $\nabla$ \\ Dr. med. Kay Schaefer, Köln}

Die Fortbildungsreisen sind für Ärzte aus Europa und den USA, die klinische Erfahrung auf dem Gebiet der Tropen- und Reisemedizin sammeln möchten. Während der 13-tägigen Rundreisen werden verschiedene Lehrkrankenhäuser, ambulante Stationen und Forschungsprojekte in Kenia, Uganda und Tansania besucht. Experten lehren am Krankenbett über die Klinik und Therapie der wichtigsten tropischen Infektionskrankheiten. Laborpraktika (mikroskopische Untersuchung von Parasiten in Blut, Stuhl und Urin) und Vorlesungen runden das medizinische Programm ab. Tropische Dermatosen spielen eine große Rolle in Ostafrika. Während der Rundreise durch Kenia sehen die Ärzte im Marigat Health Center, nicht weit vom Äquator im trockenen und sehr heißen Rift-Valley, Patienten mit diffusen, erytematösen und nodulären Hautläsionen im Gesicht. Dieses Krankheitsbild nennt man Post-Kala-Azar-dermales Leishmanoid (PKDL) und es tritt manchmal nach einer auskurierten viszeralen Leishmaniose (Anthroponose) auf, die durch Sandfliegen (Phlebotomus) übertragen wird. PKDL kann mit der PCR oder monoklonalen Antikörpern (Sensitivität $>80 \%$ ) diagnostiziert werden. Der direkte Nachweis des Erregers (Leishmania [L.] donovani) aus dem Hautausstrich erweist sich oft als schwierig. In Kenia wird die Diagnose meistens klinisch gestellt. Des Öfteren kommen dort auch Patienten mit ulzerierenden, zum Teil auch nekrotisierenden Hautläsionen mit schlechter oder später Spontanheilung in die Ambulanz. Die Ulzera sind schmerzlos und befinden sich vor allem im Gesicht oder an den unbedeckten Extremitäten. Diese Patienten leiden an der kutanen Leishmaniose. Die Diagnose erfolgt über den direkten Nachweis des Protozoen (L. major) aus dem Wundrand (Biopsie oder Punktat).

In Uganda ist die Schlafkrankheit (Afrikanische Trypanosomiasis) endemisch. In der Nähe des Viktoria-Sees nehmen die Ärzte an einer mobilen „Dermatology Clinic“ teil. Ein Fischer klagt über einen Tse-Tse-Fliegen-Stich am Oberarm, der sich in den letzten 2 Wochen entzündet hat. Die schmerzhafte Primärläsion an der Inokulationsstelle mit Schwellung der regionalen Lymphknoten muss unbedingt abgeklärt werden, da die Schlafkrankheit im fortgeschrittenen Stadium tödlich verlaufen kann. Das Punktat des Primärschankers wird im Feldlabor $(\boldsymbol{Q} \mathbf{A b b} \mathbf{5} \mathbf{5} \mathbf{a})$ mit Giemsa angefärbt und untersucht. Kurze Zeit später entdeckt der Laborassistent begeißelte Protozoen (Trypanosoma rhodesiense) unter dem Mikroskop. Der direkte Nachweis aus dem Trypanosomenschanker ist umso bedeutender, da der Blutausstrich und die Serologie (ELISA, IFAT) erst positiv werden, wenn die Parasiten über die Lymphe und das Blut den großen Kreislauf erreicht haben.

Auf Sansibar in Tansania besuchen die Ärzte das „Schistosomiasis Prevention \& Control Project“, das vom Parasitologen Dr. Khalfan geleitet wird. In dem großen Labor arbeiten 5 Assistenten an verschiedenen Mikroskopen. Sie untersuchen Stuhl- und Urinproben 

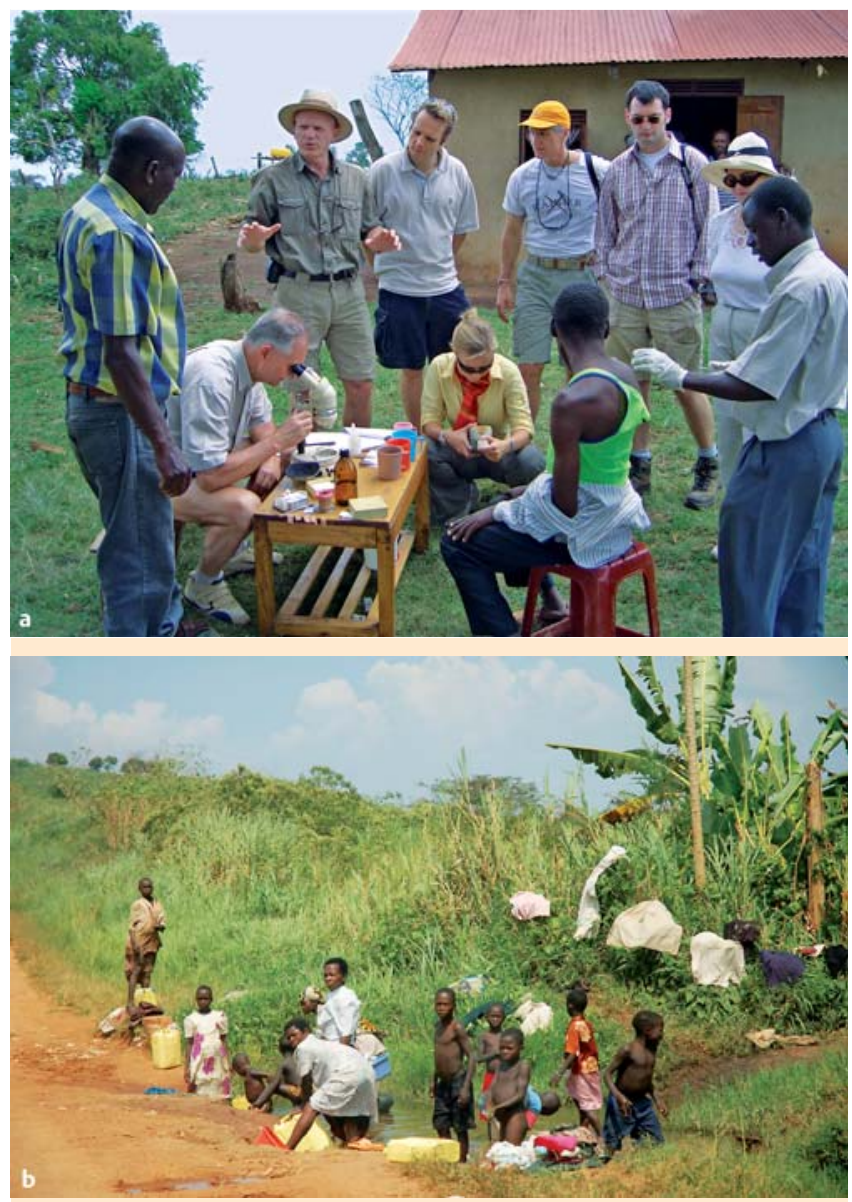

Abb. 5 a Das Punktat des Primärschankers wird im Feldlabor mit Giemsa angefärbt und untersucht. Begeißelte Protozoen (Trypanosoma rhodesiense) sind im Auflichtmikroskop erkennbar. b Habitat der Schistosomiasis (Bilharziose, Zerkarien-Infektion) auf Sansibar, Tansania. auf Parasiten. Mit Unterstützung der WHO hat sich Sansibar zum Ziel gesetzt, die Bilharziose auf der Insel im Indischen Ozean auszurotten. Hinter dem Labor ist ein kleiner Untersuchungsraum, in dem Patienten untersucht werden können. Ein Junge, nicht älter als 10 Jahre, hat ein juckendes, makulopapulöses Exanthem am ganzen Körper. Erst nach eingehender Befragung auf Kiswahili erzählt Mohammed, dass er kürzlich mit Freunden in einem kleinen Süßwassertümpel Schwimmen war ( $\boldsymbol{A} \mathbf{A b b}$. 5 b ). Die Symptome von Mohammed deuten laut Aussage von Dr. Khalfan auf eine Zerkariendermatitis hin. Während der Hautpenetration des Erregers (S. haematobium oder S. mansoni) kann es zu Pruritus, Erythem und flüchtigem makulopapulösen Exanthem kommen. In diesem frühen Stadium kann die Serologie (ELISA) schon positiv sein. Außerdem findet man oft eine massive Eosinophilie. Erst 4-10 Wochen (Präpatenzzeit) nach der Infektion kann man Eier im Urin ( $S$. haematobium) oder Stuhl (S. mansoni) nachweisen. Dr. Khalfan erinnert uns: „Ausgeprägte Erscheinungen bei Invasion von Zerkarien von nicht humanpathogenen Schistosomen in Europa und den USA können ebenfalls zu einer Zerkariendermatitis, bei uns auch Badedermatitis genannt, führen."

Da aufgrund des wachsenden Tourismus immer häufiger Patienten mit Dermatosen aus den Tropen zurückkehren, sollten deutsche Dermatologen auf diesem Gebiet klinische Erfahrungen sammeln, um oben genannte Krankheitsbilder frühzeitig erkennen zu können. Die Fortbildungsreisen nach Ostafrika gewähren eine praxisbezogene, klinische Fortbildung auf dem Gebiet der tropischen Dermatologie und sind jedem interessierten Hautarzt zu empfehlen.

Die Teilnahme schließt mit einem Zertifikat über 60 Lehrstunden auf dem Gebiet der klinischen Tropen- und Reisemedizin ab. Dies entspricht 30 Fortbildungspunkten im gesamten Ärztekammerbereich der BRD. Darüber hinaus ist ein 9-stündiger RefresherKurs für Reisemedizin, anerkannt von der Deutschen Tropenmedizinischen Gesellschaft (DTG), in die Fortbildungsreise integriert. Die nächsten Termine der tropenmedizinischen Fortbildungsreisen sind wie folgt (http://www.tropmedex.com): Kenia 17.29. 1. 2010, Uganda 14. - 26. 2. 2010, Tansania 14.-26.3. 2010. 